\title{
$m$-Berezin transform and compact operators
}

\section{Kyesook Nam, Dechao Zheng and Changyong Zhong}

\begin{abstract}
$m$-Berezin transforms are introduced for bounded operators on the Bergman space of the unit ball. The norm of the $m$-Berezin transform as a linear operator from the space of bounded operators to $L^{\infty}$ is found. We show that the $m$-Berezin transforms are commuting with each other and Lipschitz with respect to the pseudo-hyperbolic distance on the unit ball. Using the $m$-Berezin transforms we show that a radial operator in the Toeplitz algebra is compact iff its Berezin transform vanishes on the boundary of the unit ball.
\end{abstract}

\section{Introduction}

Let $B$ denote the unit ball in $n$-dimensional complex space $\mathbb{C}^{n}$ and $d z$ be normalized Lebesgue volume measure on $B$. The Bergman space $L_{a}^{2}=$ $L_{a}^{2}(B, d z)$ is the space of analytic functions $h$ on $B$ which are square-integrable with respect to Lebesgue volume measure. For $z=\left(z_{1}, \ldots, z_{n}\right) \in \mathbb{C}^{n}$, let $\langle z, w\rangle=\sum_{i=1}^{n} z_{i} \bar{w}_{i}$ and $|z|^{2}=\langle z, z\rangle$.

For $z \in B$, let $P_{z}$ be the orthogonal projection of $\mathbb{C}^{n}$ onto the subspace $[z]$ generated by $z$ and let $Q_{z}=I-P_{z}$. Then

$$
\phi_{z}(w)=\frac{z-P_{z}(w)-\left(1-|z|^{2}\right)^{1 / 2} Q_{z}(w)}{1-\langle w, z\rangle}
$$

is the automorphism of $B$ that interchanges 0 and $z$. The pseudo-hyperbolic metric on $B$ is defined as $\rho(z, w)=\left|\phi_{z}(w)\right|$.

The reproducing kernel in $L_{a}^{2}$ is given by

$$
K_{z}(w)=\frac{1}{(1-\langle w, z\rangle)^{n+1}},
$$

for $z, w \in B$ and the normalized reproducing kernel $k_{z}$ is $K_{z}(w) /\left\|K_{z}(\cdot)\right\|_{2}$.

2000 Mathematics Subject Classification: 47B35, $47 \mathrm{~B} 38$.

Keywords: $m$-Berezin transforms, Toeplitz operators. 
If $\langle\cdot, \cdot\rangle$ denotes the inner product in $L^{2}$, then $\left\langle h, K_{z}\right\rangle=h(z)$, for every $h \in L_{a}^{2}$ and $z \in B$. The fundamental property of the reproducing kernel $K_{z}(w)$ plays an important role in this paper:

$$
K_{z}(w)=\overline{k_{\lambda}(z)} K_{\phi_{\lambda}(z)}\left(\phi_{\lambda}(w)\right) k_{\lambda}(w) .
$$

Given $f \in L^{\infty}$, the Toeplitz operator $T_{f}$ is defined on $B$ by $T_{f} h=P(f h)$ where $P$ denotes the orthogonal projection $P$ of $L^{2}$ onto $L_{a}^{2}$.

Let $\mathfrak{L}\left(L_{a}^{2}\right)$ be the algebra of bounded operators on $L_{a}^{2}$. The Toeplitz algebra $\mathfrak{T}\left(L^{\infty}\right)$ is the closed subalgebra of $\mathfrak{L}\left(L_{a}^{2}\right)$ generated by $\left\{T_{f}: f \in L^{\infty}\right\}$.

For $z \in B$, let $U_{z}$ be the unitary operator given by

$$
U_{z} f=\left(f \circ \phi_{z}\right) \cdot J \phi_{z}
$$

where $J \phi_{z}=(-1)^{n} k_{z}$. For $S \in \mathfrak{L}\left(L_{a}^{2}\right)$, set

$$
S_{z}=U_{z} S U_{z} \text {. }
$$

Since $U_{z}$ is a selfadjoint unitary operator on $L^{2}$ and $L_{a}^{2}, U_{z} T_{f} U_{z}=T_{f \circ \phi_{z}}$ for every $f \in L^{\infty}$.

Let $\mathcal{T}$ denote the class of trace operators on $L_{a}^{2}$. For $T \in \mathcal{T}$, we will denote the trace of $T$ by $\operatorname{tr}[T]$ and let $\|T\|_{C_{1}}$ denote the $C_{1}$ norm of $T$ given by $([12])$

$$
\|T\|_{C_{1}}=\operatorname{tr}\left[\sqrt{T^{*} T}\right] .
$$

Suppose $f$ and $g$ are in $L_{a}^{2}$. Consider the operator $f \otimes g$ on $L_{a}^{2}$ defined by

$$
(f \otimes g) h=\langle h, g\rangle f,
$$

for $h \in L_{a}^{2}$. It is easily proved that $f \otimes g$ is in $\mathcal{T}$ and with norm equal to $\|f \otimes g\|_{C_{1}}=\|f\|_{2}\|g\|_{2}$ and

$$
\operatorname{tr}[f \otimes g]=\langle f, g\rangle .
$$

For a nonnegative integer $m$, the $m$-Berezin transform of an operator $S \in \mathfrak{L}\left(L_{a}^{2}\right)$ is defined by

$$
\begin{aligned}
B_{m} S(z) & =C_{n}^{m+n} \operatorname{tr}\left[S_{z}\left(\sum_{|k|=0}^{m} C_{m, k} \frac{n ! k !}{(n+|k|) !} \frac{u^{k}}{\left\|u^{k}\right\|} \otimes \frac{u^{k}}{\left\|u^{k}\right\|}\right)\right] \\
& =C_{n}^{m+n} \operatorname{tr}\left[S_{z}\left(\sum_{|k|=0}^{m} C_{m, k} u^{k} \otimes u^{k}\right)\right]
\end{aligned}
$$

where $k=\left(k_{1}, \cdots, k_{n}\right) \in N^{n}, N$ is the set of nonnegative integers, $|k|=$ $\sum_{i=0}^{n} k_{i}, u^{k}=u_{1}^{k_{1}} \cdots u_{n}^{k_{n}}, k !=k_{1} ! \cdots k_{n}$ !,

$$
C_{n}^{m+n}=\left(\begin{array}{c}
m+n \\
n
\end{array}\right) \quad \text { and } \quad C_{m, k}=C_{|k|}^{m}(-1)^{|k|} \frac{|k| !}{k_{1} ! \cdots k_{n} !} .
$$


Clearly, $B_{m}: \mathfrak{L}\left(L_{a}^{2}\right) \rightarrow L^{\infty}$ is a bounded linear operator, the norm of $B_{m}$ will be given.

Given $f \in L^{\infty}$, define

$$
B_{m}(f)(z)=B_{m}\left(T_{f}\right)(z) .
$$

$B_{m}(f)(z)$ equals the nice formula in [1]:

$$
B_{m}(f)(z)=\int_{B} f \circ \phi_{z}(u) d \nu_{m}(u)
$$

for $z \in B$ where $d \nu_{m}(u)=C_{n}^{m+n}\left(1-|u|^{2}\right)^{m} d u$.

Berezin first introduced the Berezin transform $B_{0}(S)$ of bounded operators $S$ and the $m$-Berezin transform of functions in [5]. Because the Berezin transform encodes operator-theoretic information in function-theory in a striking but somewhat impenetrable way, the Berezin transform $B_{0}(S)$ has found useful applications in studying operators of "function-theoretic significance" on function spaces ([2], [3], [4], [6], [7], [11], and [15]). Suárez [16] introduced $m$-Berezin transforms of bounded operators on the Bergman space of the unit disk. We will show that our $m$-Berezin transform coincides with the one defined in [16] on the unit disk $D$ by means of an integral representation of $m$-Berezin transform. The integral representation shows that many useful properties of the $m$-Berezin transforms inherit from the identity (1.1) of the reproducing kernel. On the unit ball, some useful properties of the $m$-Berezin transforms of functions were obtained by Ahern, Flores and Rudin [1]. Recently, Coburn [10] proved that $B_{0}(S)$ is Lipschitz with respect to the pseudo-hyperbolic distance $\rho(z, w)$. In this paper, we will show that $B_{m} S(z)$ is Lipschitz with respect to pseudo-hyperbolic distance $\rho(z, w)$. We will show that the $m$-Berezin transforms $B_{m}$ are invariant under the Mobious transform,

$$
B_{m}\left(S_{z}\right)=\left(B_{m} S\right) \circ \phi_{z}
$$

and commuting with each other,

$$
B_{j}\left(B_{m} S\right)(z)=B_{m}\left(B_{j} S\right)(z)
$$

for any nonnegative integers $j$ and $m$. Properties (1.3) and (1.4) were obtained for $S=T_{f}$ in [1] and for operators $S$ on the Bergman space of the unit disk [16].

A common intuition is that for operators on the Bergman space $L_{a}^{2}$ "closely associated with function theory", compactness is equivalent to having vanishing Berezin transform on the boundary of the unit ball $B$. On the 
unit disk, Axler and Zheng [2] showed that if the operator $S$ equals the finite sum of finite products of Toeplitz operators with bounded symbols then $S$ is compact if and only if $B_{0}(S)(z) \rightarrow 0$ as $z \rightarrow \partial D$. Englis extended this result to the unit ball even the bounded symmetric domains [11]. But the problem remains open whether the result is true if $S$ is in the Toeplitz algebra. Recently, Suárez [17] solved the problem for radial operator $S$ on the unit disk via the $m$-Berezin transform. Using the $m$-Berezin transform, we will show that for a radial operator $S$ in the Toeplitz algebra on the unit ball, $S$ is compact iff $B_{0} S(z) \rightarrow 0$ as $|z| \rightarrow 1$.

Throughout the paper $C(m, n)$ will denote constant depending only on $m$ and $n$, which may change at each occurrence.

The authors thank the referees for their suggestions.

\section{2. $m$-Berezin transform}

In this section we will show some useful properties of the $m$-Berezin transform. First we give an integral representation of the $m$-Berezin transform $B_{m}(S)$. For $z \in B$ and a nonnegative integer $m$, let

$$
K_{z}^{m}(u)=\frac{1}{(1-\langle u, z\rangle)^{m+n+1}}, \quad u \in B .
$$

For $u, \lambda \in B$, we can easily see that

$$
\sum_{|k|=0}^{m} C_{m, k} u^{k} \overline{\lambda^{k}}=(1-\langle u, \lambda\rangle)^{m} .
$$

Proposition 2.1 Let $S \in \mathfrak{L}\left(L_{a}^{2}\right), m \geq 0$ and $z \in B$. Then

$$
\begin{aligned}
B_{m} S(z) & =C_{n}^{m+n}\left(1-|z|^{2}\right)^{m+n+1} \times \\
& \int_{B} \int_{B}(1-\langle u, \lambda\rangle)^{m} K_{z}^{m}(u) \overline{K_{z}^{m}(\lambda) S^{*} K_{\lambda}(u)} d u d \lambda .
\end{aligned}
$$

Proof. For $\lambda \in B$, the definition of $B_{m}$ implies

$$
\begin{aligned}
B_{m} S(z) & =C_{n}^{m+n} \sum_{|k|=0}^{m} C_{m, k}\left\langle S_{z} \lambda^{k}, \lambda^{k}\right\rangle \\
& =C_{n}^{m+n} \sum_{|k|=0}^{m} C_{m, k} \int_{B} S\left(\phi_{z}^{k} k_{z}\right)(\lambda) \overline{\phi_{z}^{k}(\lambda) k_{z}(\lambda)} d \lambda \\
& =C_{n}^{m+n} \sum_{|k|=0}^{m} C_{m, k} \int_{B} \int_{B} \phi_{z}^{k}(u) k_{z}(u) \overline{\phi_{z}^{k}(\lambda) k_{z}(\lambda) S^{*} K_{\lambda}(u)} d u d \lambda
\end{aligned}
$$

where the last equality holds by $S\left(\phi_{z}^{k} k_{z}\right)(\lambda)=\left\langle S\left(\phi_{z}^{k} k_{z}\right), K_{\lambda}\right\rangle=\left\langle\phi_{z}^{k} k_{z}, S^{*} K_{\lambda}\right\rangle$. 
Using (2.1) and (1.1), (2.2) equals

$$
\begin{aligned}
& C_{n}^{m+n} \int_{B} \int_{B}\left(1-\left\langle\phi_{z}(u), \phi_{z}(\lambda)\right\rangle\right)^{m} k_{z}(u) \overline{k_{z}(\lambda) S^{*} K_{\lambda}(u)} d u d \lambda \\
& =C_{n}^{m+n} \int_{B} \int_{B}\left(\frac{k_{z}(u) \overline{k_{z}(\lambda)}}{K_{\lambda}(u)}\right)^{m /(n+1)} k_{z}(u) \overline{k_{z}(\lambda) S^{*} K_{\lambda}(u)} d u d \lambda \\
& =C_{n}^{m+n}\left(1-|z|^{2}\right)^{m+n+1} \int_{B} \int_{B}(1-\langle u, \lambda\rangle)^{m} K_{z}^{m}(u) \overline{K_{z}^{m}(\lambda) S^{*} K_{\lambda}(u)} d u d \lambda
\end{aligned}
$$

as desired.

Proposition 2.2 gives another form of $B_{m}$.

Proposition 2.2 Let $S \in \mathfrak{L}\left(L_{a}^{2}\right), m \geq 0$ and $z \in B$. Then

$$
B_{m} S(z)=C_{n}^{m+n}\left(1-|z|^{2}\right)^{m+n+1} \sum_{|k|=0}^{m} C_{m, k}\left\langle S\left(u^{k} K_{z}^{m}\right), u^{k} K_{z}^{m}\right\rangle .
$$

Proof. Since

$$
\begin{aligned}
\int_{B} \int_{B}(1 & -\langle u, \lambda\rangle)^{m} K_{z}^{m}(u) \overline{K_{z}^{m}(\lambda) S^{*} K_{\lambda}(u)} d u d \lambda \\
& =\sum_{|k|=0}^{m} C_{m, k} \int_{B} \int_{B} u^{k} \overline{\lambda^{k}} K_{z}^{m}(u) \overline{K_{z}^{m}(\lambda) S^{*} K_{\lambda}(u)} d u d \lambda \\
& =\sum_{|k|=0}^{m} C_{m, k} \int_{B} S\left(u^{k} K_{z}^{m}\right)(\lambda) \overline{\lambda^{k} K_{z}^{m}(\lambda)} d \lambda
\end{aligned}
$$

Proposition 2.1 implies (2.3).

For $n=1$, the right hand side of (2.3) was used by Suárez in [16] to define the $m$-Berezin transforms on the unit disk.

Recall that given $f \in L^{\infty}$, define

$$
B_{m}(f)(z)=B_{m}\left(T_{f}\right)(z) .
$$

The following proposition gives a nice formula of $B_{m}(f)(z)$. Let $d \nu_{m}(u)=$ $C_{n}^{m+n}\left(1-|u|^{2}\right)^{m} d u$.

Proposition 2.3 Let $z \in B$ and $f \in L^{\infty}$. Then

$$
B_{m}(f)(z)=\int_{B} f \circ \phi_{z}(u) d \nu_{m}(u)
$$


Proof. By the change of variables, Theorem 2.2.2 in [14] and (2.3), we have

$$
\begin{aligned}
& \int_{B} f \circ \phi_{z}(u) d \nu_{m}(u) \\
& \quad=C_{n}^{m+n} \int_{B} f(u)\left(\frac{\left(1-|z|^{2}\right)\left(1-|u|^{2}\right)}{|1-\langle u, z\rangle|^{2}}\right)^{m}\left(\frac{\left(1-|z|^{2}\right)}{|1-\langle u, z\rangle|^{2}}\right)^{n+1} d u \\
& \quad=C_{n}^{m+n}\left(1-|z|^{2}\right)^{m+n+1} \sum_{|k|=0}^{m} C_{m, k} \int_{B} f(u)\left|u^{k}\right|^{2}\left|K_{z}^{m}(u)\right|^{2} d u \\
& =C_{n}^{m+n}\left(1-|z|^{2}\right)^{m+n+1} \sum_{|k|=0}^{m} C_{m, k}\left\langle T_{f}\left(u^{k} K_{z}^{m}\right), u^{k} K_{z}^{m}\right\rangle=B_{m}\left(T_{f}\right)(z) .
\end{aligned}
$$

The proof is complete.

The formula in the above proposition was used in [1] to define the $m$ Berezin transform of functions $f$.

Clearly, (1.2) gives $\left\|B_{m} S\right\|_{\infty} \leq C(m, n)\left\|S_{z}\right\|=C(m, n)\|S\|$ for $S \in$ $\mathfrak{L}\left(L_{a}^{2}\right)$. Thus, $B_{m}: \mathfrak{L}\left(L_{a}^{2}\right) \rightarrow L^{\infty}$ is a bounded linear operator. The following theorem gives the norm of $B_{m}$.

Theorem 2.4 Let $m \geq 0$. Then

$$
\left\|B_{m}\right\|=C_{n}^{m+n} \sum_{|k|=0}^{m}\left|C_{m, k}\right| \frac{n ! k !}{(n+|k|) !} .
$$

Proof. From [8], we have the duality result $\mathfrak{L}\left(L_{a}^{2}\right)=\mathcal{T}^{*}$. So, the definition of $B_{m}$ gives the norm of $B_{m}$. In fact,

$$
\begin{aligned}
\left\|B_{m}\right\| & =\left\|C_{n}^{m+n} \sum_{|k|=0}^{m} C_{m, k} \frac{n ! k !}{(n+|k|) !} \frac{u^{k}}{\left\|u^{k}\right\|} \otimes \frac{u^{k}}{\left\|u^{k}\right\|}\right\|_{C_{1}} \\
& =C_{n}^{m+n} \sum_{|k|=0}^{m}\left|C_{m, k}\right| \frac{n ! k !}{(n+|k|) !}
\end{aligned}
$$

as desired.

The Mobius map $\phi_{z}(w)$ has the following property ([14]):

$$
\phi_{z}^{\prime}(0)=-\left(1-|z|^{2}\right) P_{z}-\left(1-|z|^{2}\right)^{1 / 2} Q_{z} .
$$

To show that $m$-Berezin transforms are Lipschitz with respect to the pseudohyperbolic distance we need the following lemmas.

For $z, w \in \mathbb{C}^{n}, z \hat{\otimes} w$ on $\mathbb{C}^{n}$ is defined by $(z \hat{\otimes} w) \lambda=\langle\lambda, w\rangle z$. 
Lemma 2.5 Let $z, w \in B$ and $\lambda=\phi_{z}(w)$. Then

$$
\phi_{z}^{\prime}(w)=(1-\langle\lambda, z\rangle)(I-\lambda \hat{\otimes} z)\left[\phi_{z}^{\prime}(0)\right]^{-1} .
$$

Proof. Suppose that $P_{z}$ and $Q_{z}$ have the matrix representations as $\left(\left(P_{z}\right)_{i j}\right)$ and $\left(\left(Q_{z}\right)_{i j}\right)$ under the standard base of $\mathbb{C}^{n}$, respectively. In fact,

$$
\left(P_{z}\right)_{i j}=\frac{z_{i} \bar{z}_{j}}{|z|^{2}} \quad \text { if } \quad z \neq 0 .
$$

Let $\left(a_{i j}(w)\right)=\phi_{z}^{\prime}(w)$. Write $\phi_{z}(w)=\left(f_{1}(w), \ldots, f_{n}(w)\right)$. Then

$$
a_{i j}(w)=\frac{\partial f_{i}}{\partial w_{j}}(w)
$$

Noting that

$$
f_{i}(w)=\frac{z_{i}-\left(P_{z} w\right)_{i}-\left(1-|z|^{2}\right)^{1 / 2}\left(Q_{z} w\right)_{i}}{1-\langle w, z\rangle}
$$

we have

$$
\begin{aligned}
a_{i j}(w) & =\frac{\left(z_{i}-\left(P_{z} w\right)_{i}-\left(1-|z|^{2}\right)^{1 / 2}\left(Q_{z} w\right)_{i}\right) \bar{z}_{j}}{(1-\langle w, z\rangle)^{2}}-\frac{\left(P_{z}\right)_{i j}+\left(1-|z|^{2}\right)^{1 / 2}\left(Q_{z}\right)_{i j}}{1-\langle w, z\rangle} \\
& =\frac{f_{i}(w) \bar{z}_{j}}{1-\langle w, z\rangle}-\frac{\left(P_{z}\right)_{i j}+\left(1-|z|^{2}\right)^{1 / 2}\left(Q_{z}\right)_{i j}}{1-\langle w, z\rangle} .
\end{aligned}
$$

Let $\lambda=\phi_{z}(w)$. The above equality becomes

$$
a_{i j}(w)=\frac{\lambda_{i} \bar{z}_{j}-\left(\left(P_{z}\right)_{i j}+\left(1-|z|^{2}\right)^{1 / 2}\left(Q_{z}\right)_{i j}\right)}{1-\langle w, z\rangle}
$$

Thus

$$
\phi_{z}^{\prime}(w)=\frac{\lambda \hat{\otimes} z-\left(P_{z}+\left(1-|z|^{2}\right)^{1 / 2} Q_{z}\right)}{1-\langle w, z\rangle} .
$$

From Theorem 2.2.5 in [14], we have

$$
\frac{1}{1-\langle w, z\rangle}=\frac{1-\langle\lambda, z\rangle}{1-|z|^{2}} .
$$

Thus (2.4) implies

$$
\begin{aligned}
\phi_{z}^{\prime}(w) \phi_{z}^{\prime}(0) & =\frac{-\left(1-|z|^{2}\right) \lambda \hat{\otimes} z+\left(1-|z|^{2}\right) P_{z}+\left(1-|z|^{2}\right) Q_{z}}{1-\langle w, z\rangle} \\
& =\frac{\left(1-|z|^{2}\right)(-\lambda \hat{\otimes} z+I)}{1-\langle w, z\rangle} \\
& =(1-\langle\lambda, z\rangle)(I-\lambda \hat{\otimes} z)
\end{aligned}
$$

where the first equality follows from $P_{z} Q_{z}=Q_{z} P_{z}=0, P_{z} z=z$, and $Q_{z} z=0$. The proof is complete. 
Lemma 2.6 Suppose $|z|>1 / 2$ and $|w|>1 / 2$. If $\left|\phi_{z}(w)\right| \leq \epsilon<1 / 2$, then

$$
\left\|P_{z}-P_{w}\right\| \leq 50 \epsilon\left(1-|z|^{2}\right)^{1 / 2} .
$$

Proof. First we will get the estimate of the distance between $z$ and $w$. Since $\left|\phi_{z}(w)\right| \leq \epsilon<1 / 2, w$ is in the ellipsoid:

$$
\phi_{z}(\epsilon B)=\left\{w \in B: \frac{\left|P_{z} w-c\right|^{2}}{\epsilon^{2} \rho^{2}}+\frac{\left|Q_{z} w\right|^{2}}{\epsilon^{2} \rho}<1\right\}
$$

with center $c=\frac{\left(1-\epsilon^{2}\right) z}{\left(1-\epsilon^{2}|z|^{2}\right)}$ and $\rho=\frac{1-|z|^{2}}{1-\epsilon^{2}|z|^{2}}$. Noting that $|z|>1 / 2$ and $\epsilon<1 / 2$, we have $\rho \leq 2\left(1-|z|^{2}\right)$. Thus

$$
\left|Q_{z} w\right|^{2} \leq \epsilon^{2} \rho \leq 2 \epsilon^{2}\left(1-|z|^{2}\right), \quad\left|P_{z} w-c\right| \leq \epsilon \rho \leq 2 \epsilon\left(1-|z|^{2}\right)
$$

and

$$
|z-c| \leq \frac{\epsilon^{2}\left(1-|z|^{2}\right)}{\left(1-\epsilon^{2}|z|^{2}\right)} \leq 2 \epsilon^{2}\left(1-|z|^{2}\right)
$$

So, we have

$$
\left|P_{z} w-z\right| \leq\left|P_{z} w-c\right|+|z-c| \leq 3 \epsilon\left(1-|z|^{2}\right) .
$$

Because $I=P_{z}+Q_{z}$ and $P_{z} Q_{z}=0$, writing

$$
(z-w)=P_{z}(z-w)+Q_{z}(z-w),
$$

we have

$$
\begin{aligned}
|z-w|^{2} & =\left|P_{z}(z-w)\right|^{2}+\left|Q_{z}(z-w)\right|^{2} \\
& =\left|P_{z} w-z\right|^{2}+\left|Q_{z} w\right|^{2} \\
& \leq 11 \epsilon^{2}\left(1-|z|^{2}\right) .
\end{aligned}
$$

Noting that

$\frac{z}{|z|} \hat{\otimes} \frac{z}{|z|}=\frac{(z-w)}{|z|} \hat{\otimes} \frac{z}{|z|}+\frac{w}{|z|} \hat{\otimes} \frac{(z-w)}{|z|}+\left[\left(\frac{1}{|z|^{2}}-\frac{1}{|w|^{2}}\right) w\right] \hat{\otimes} w+\frac{w}{|w|} \hat{\otimes} \frac{w}{|w|}$,

we have

$$
P_{z}-P_{w}=\frac{(z-w)}{|z|} \hat{\otimes} \frac{z}{|z|}+\frac{w}{|z|} \hat{\otimes} \frac{(z-w)}{|z|}+\left[\left(\frac{1}{|z|^{2}}-\frac{1}{|w|^{2}}\right) w\right] \hat{\otimes} w
$$

to obtain

$$
\begin{aligned}
\left\|P_{z}-P_{w}\right\| & \leq \frac{|z-w|}{|z|}+\frac{2|z-w|}{|z|}+\frac{\left.|| z\right|^{2}-|w|^{2} \mid}{|z|^{2}} \\
& \leq 2|z-w|+4|z-w|+8|z-w| \\
& \leq 14 \sqrt{11} \epsilon\left(1-|z|^{2}\right)^{1 / 2} \\
& \leq 50 \epsilon\left(1-|z|^{2}\right)^{1 / 2}
\end{aligned}
$$

where the last inequality holds by (2.5). 
For given $z, w \in B$, set $A(z, w)=-\left(1-|z|^{2}\right) P_{w}-\left(1-|z|^{2}\right)^{1 / 2} Q_{w}$.

Lemma 2.7 Suppose $|z|>1 / 2$ and $|w|>1 / 2$. If $\left|\phi_{z}(w)\right| \leq \epsilon<1 / 2$, then

$$
\left\|\phi_{z}^{\prime}(0)-A(z, w)\right\| \leq 150 \epsilon\left(1-|z|^{2}\right) .
$$

Proof. Using (2.4), we have

$$
\begin{aligned}
\left\|\phi_{z}^{\prime}(0)-A(z, w)\right\| & =\left\|\left(1-|z|^{2}\right)\left(P_{w}-P_{z}\right)+\left(1-|z|^{2}\right)^{1 / 2}\left(P_{z}-P_{w}\right)\right\| \\
& \leq 3\left(1-|z|^{2}\right)^{1 / 2}\left\|P_{z}-P_{w}\right\| \\
& \leq 150 \epsilon\left(1-|z|^{2}\right)
\end{aligned}
$$

as desired. The last inequality follows from Lemma 2.6.

Let $\mathfrak{U}(n)$ be the group of $n \times n$ complex unitary matrices.

Lemma 2.8 Let $z, w \in B$. Then $U_{z} U_{w}=V_{\mathcal{U}} U_{\phi_{w}}(z)$ where

$$
\left(V_{\mathcal{U}} f\right)(u)=f(\mathcal{U} u) \operatorname{det} \mathcal{U}
$$

for $f \in L_{a}^{2}$ and $\mathcal{U}=\phi_{\phi_{w}(z)} \circ \phi_{w} \circ \phi_{z}$ satisfying

$$
\|I+\mathcal{U}\| \leq C(n) \rho(z, w) .
$$

Proof. The map $\phi_{\phi_{w}(z)} \circ \phi_{w} \circ \phi_{z}$ is an automorphism of $B$ that fixes 0, hence it is unitary by the Cartan theorem in [14]. Thus $\phi_{w} \circ \phi_{z}=\phi_{\phi_{w}(z)} \circ \mathcal{U}$ for some $\mathcal{U} \in \mathfrak{U}(n)$. Since $\phi_{w}$ is an involution, we have

$$
\begin{aligned}
& U_{z} U_{w} f(u)=\left(f \circ \phi_{w} \circ \phi_{z}\right)(u) J \phi_{w}\left(\phi_{z}(u)\right) J \phi_{z}(u) \\
& =\left(f \circ \phi_{\phi_{w}(z)}\right)(\mathcal{U} u) J \phi_{w}\left(\phi_{w} \circ \phi_{\phi_{w}(z)}(\mathcal{U} u)\right) J \phi_{w}\left(\phi_{\phi_{w}(z)}(\mathcal{U} u)\right) J \phi_{\phi_{w}(z)}(\mathcal{U} u) \operatorname{det} \mathcal{U} \\
& =\left(f \circ \phi_{\phi_{w}(z)}\right)(\mathcal{U} u) J \phi_{\phi_{w}(z)}(\mathcal{U} u) \operatorname{det} \mathcal{U} \\
& =V_{\mathcal{U}} U_{\phi_{w}(z)} f(u)
\end{aligned}
$$

as desired.

Now we will show that

$$
\|I+\mathcal{U}\| \leq C(n) \rho(z, w) .
$$

Noting that $\mathcal{U}$ is continuous for $|z| \leq 1 / 2$ and $|w| \leq 1 / 2$, we need only to prove

$$
\|I+\mathcal{U}\| \leq 20000 \rho(z, w)
$$

for $|z|>1 / 2,|w|>1 / 2$ and $\left|\phi_{w}(z)\right|<1 / 2$. Let $\lambda=\phi_{w}(z)$. Then $|\lambda|=$ $\rho(z, w)$ and $z=\phi_{w}(\lambda)$. Since

$$
\phi_{w} \circ \phi_{z}(u)=\phi_{\lambda}(\mathcal{U} u)
$$

taking derivatives both sides of the above equations and using the chain rule give

$$
\phi_{w}^{\prime}\left(\phi_{z}(u)\right) \phi_{z}^{\prime}(u)=\phi_{\lambda}^{\prime}(\mathcal{U} u) \mathcal{U}
$$


Letting $u=0$, the above equality gives

$$
\mathcal{U}=\left[\phi_{\lambda}^{\prime}(0)\right]^{-1} \phi_{w}^{\prime}(z) \phi_{z}^{\prime}(0) .
$$

By Lemma 2.5, write

$$
\begin{aligned}
\mathcal{U}+I= & {\left[\phi_{\lambda}^{\prime}(0)\right]^{-1}(1-\langle\lambda, w\rangle)(I-\lambda \hat{\otimes} w)\left[\phi_{w}^{\prime}(0)\right]^{-1} \phi_{z}^{\prime}(0)+I } \\
= & {\left[\phi_{\lambda}^{\prime}(0)\right]^{-1}(1-\langle\lambda, w\rangle)(I-\lambda \hat{\otimes} w)\left[\phi_{w}^{\prime}(0)\right]^{-1}\left[\phi_{z}^{\prime}(0)-A(z, w)\right] } \\
& \quad+\left(\left[\phi_{\lambda}^{\prime}(0)\right]^{-1}(1-\langle\lambda, w\rangle)(I-\lambda \hat{\otimes} w)\left[\phi_{w}^{\prime}(0)\right]^{-1} A(z, w)+I\right) \\
:= & I_{1}+I_{2} .
\end{aligned}
$$

By Lemma 2.7, we have

$$
\begin{aligned}
\left\|I_{1}\right\| & \leq\left\|\left[\phi_{\lambda}^{\prime}(0)\right]^{-1}\right\||1-\langle\lambda, w\rangle|\|I-\lambda \hat{\otimes} w\|\left\|\left[\phi_{w}^{\prime}(0)\right]^{-1}\right\|\left\|\phi_{z}^{\prime}(0)-A(z, w)\right\| \\
& \leq 4 \times 2 \times 2 \times \frac{3}{\left(1-|w|^{2}\right)}\left[150|\lambda|\left(1-|z|^{2}\right)\right] .
\end{aligned}
$$

Theorem 2.2.2 in [14] leads to

$$
\frac{1-|z|^{2}}{1-|w|^{2}}=\frac{1-|\lambda|^{2}}{|1-\langle\lambda, w\rangle|^{2}}
$$

Thus

$$
\left\|I_{1}\right\| \leq 4 \times 2 \times 2 \times 3 \times 2 \times 150|\lambda|=14400|\lambda| .
$$

Also, we have

$$
\left|1-\frac{\left(1-|z|^{2}\right)^{1 / 2}}{\left(1-|w|^{2}\right)^{1 / 2}}\right| \leq\left|1-\frac{1-|z|^{2}}{1-|w|^{2}}\right| \leq 32|\lambda| .
$$

Hence, we get

$$
\left\|I-\frac{1-|z|^{2}}{1-|w|^{2}} P_{w}-\frac{\left(1-|z|^{2}\right)^{1 / 2}}{\left(1-|w|^{2}\right)^{1 / 2}} Q_{w}\right\| \leq 32|\lambda| .
$$

On the other hand, clearly,

$$
\left\|\left[\phi_{\lambda}^{\prime}(0)\right]^{-1}+I\right\| \leq 4|\lambda|, \quad|(1-\langle\lambda, w\rangle)-1| \leq|\lambda|
$$

and

$$
\|(I-\lambda \hat{\otimes} w)-I\| \leq|\lambda| .
$$

These give

$$
\left\|I+\left[\phi_{\lambda}^{\prime}(0)\right]^{-1}(1-\langle\lambda, w\rangle)(I-\lambda \hat{\otimes} w)\right\| \leq 16|\lambda| .
$$


Hence, we have

$$
\begin{aligned}
\left\|I_{2}\right\| & \leq \|\left[\phi_{\lambda}^{\prime}(0)\right]^{-1}(1-\langle\lambda, w\rangle)(I-\lambda \hat{\otimes} w)\left[\phi_{w}^{\prime}(0)\right]^{-1} A(z, w) \\
& -\left[\phi_{\lambda}^{\prime}(0)\right]^{-1}(1-\langle\lambda, w\rangle)(I-\lambda \hat{\otimes} w) \| \\
& +\left\|\left[\phi_{\lambda}^{\prime}(0)\right]^{-1}(1-\langle\lambda, w\rangle)(I-\lambda \hat{\otimes} w)+I\right\| \\
\leq \| & {\left[\phi_{\lambda}^{\prime}(0)\right]^{-1}(1-\langle\lambda, w\rangle)(I-\lambda \hat{\otimes} w)\|\| I-\frac{1-|z|^{2}}{1-|w|^{2}} P_{w}-\frac{\left(1-|z|^{2}\right)^{1 / 2}}{\left(1-|w|^{2}\right)^{1 / 2}} Q_{w} \| } \\
& +16|\lambda| \\
\leq & 42 \times 2 \times 32|\lambda|+16|\lambda|<600|\lambda| .
\end{aligned}
$$

Combining the above estimates we conclude that

$$
\|\mathcal{U}+I\| \leq 14400|\lambda|+600|\lambda|<20000|\lambda|
$$

Theorem 2.9 Let $S \in \mathfrak{L}\left(L_{a}^{2}\right), m \geq 0$ and $z \in B$. Then $B_{m} S_{z}=\left(B_{m} S\right) \circ \phi_{z}$.

Proof. Proposition 2.2 and (1.2) give

$$
B_{m} S_{z}(0)=C_{n}^{m+n} \sum_{|k|=0}^{m} C_{m, k}\left\langle S_{z} u^{k}, u^{k}\right\rangle=B_{m} S(z)=\left(B_{m} S\right) \circ \phi_{z}(0) .
$$

For any $w \in B$, Proposition 2.1 and Lemma 2.8 imply

$$
\begin{aligned}
\left(B_{m} S_{z}\right) \circ \phi_{w}(0) & =B_{m}\left(\left(S_{z}\right)_{w}\right)(0) \\
& =C_{n}^{m+n} \int_{B} \int_{B}(1-\langle u, \lambda\rangle)^{m} \overline{U_{w} U_{z} S^{*} U_{z} U_{w} K_{\lambda}(u)} d u d \lambda \\
& =C_{n}^{m+n} \int_{B} \int_{B}(1-\langle u, \lambda\rangle)^{m} \overline{V_{\mathcal{U}} U_{\phi_{z}(w)} S^{*} U_{\phi_{z}(w)} V_{\mathcal{U}}^{*} K_{\lambda}(u)} d u d \lambda \\
& =B_{m} S_{\phi_{z}(w)}(0)
\end{aligned}
$$

where $V_{\mathcal{U}}$ is in Lemma 2.8. Thus, $B_{m} S_{z}(w)=\left(B_{m} S\right) \circ \phi_{z}(w)$.

Lemma 2.10 Let $S \in \mathfrak{L}\left(L_{a}^{2}\right), m \geq 1$ and $z \in B$. Then

$$
B_{m} S(z)=\frac{m+n}{m} B_{m-1}\left(S-\sum_{i=1}^{n} T_{\overline{\left(\phi_{z}\right)_{i}}} S T_{\left(\phi_{z}\right)_{i}}\right)(z)
$$

where $\left(\phi_{z}\right)_{i}$ is $i$-th variable of $\phi_{z}$. 
Proof. By Theorem 2.9, we just need to show that

$$
B_{m} S(0)=\frac{m+n}{m} B_{m-1}\left(S-\sum_{i=1}^{n} T_{\overline{u_{i}}} S T_{u_{i}}\right)(0) .
$$

Using Proposition 2.1 and (2.1), we get

$$
\begin{aligned}
& B_{m} S(0)=C_{n}^{m+n} \int_{B} \int_{B}(1-\langle u, \lambda\rangle)^{m} \overline{S^{*} K_{\lambda}(u)} d u d \lambda \\
& =\frac{m+n}{m} B_{m-1} S(0)-C_{n}^{m+n} \sum_{i=1}^{n} \sum_{|k|=0}^{m-1} C_{m-1, k} \int_{B} \int_{B} u_{i} \overline{\lambda_{i}} u^{k} \overline{\lambda^{k} S^{*} K_{\lambda}(u)} d u d \lambda \\
& =\frac{m+n}{m} B_{m-1} S(0)-C_{n}^{m+n} \sum_{i=1}^{n} \sum_{|k|=0}^{m-1} C_{m-1, k} \int_{B} S\left(u^{k} u_{i}\right)(\lambda) \overline{\lambda^{k} \lambda_{i}} d \lambda \\
& =\frac{m+n}{m} B_{m-1} S(0)-C_{n}^{m+n} \sum_{i=1}^{n} \sum_{|k|=0}^{m-1} C_{m-1, k}\left\langle S T_{u_{i}}\left(u^{k}\right), T_{u_{i}}\left(u^{k}\right)\right\rangle
\end{aligned}
$$

as desired.

For $m=0$, the following result was obtained in [10].

Theorem 2.11 Let $S \in \mathfrak{L}\left(L_{a}^{2}\right)$ and $m \geq 0$. Then there exists a constant $C(m, n)>0$ such that

$$
\left|B_{m} S(z)-B_{m} S(w)\right|<C(m, n)\|S\| \rho(z, w) .
$$

Proof. We will prove this theorem by induction on $m$. If $m=0,(1.2)$ gives

$$
\begin{aligned}
\left|B_{0} S(z)-B_{0} S(w)\right| & =\left|\operatorname{tr}\left[S_{z}(1 \otimes 1)\right]-\operatorname{tr}\left[S_{w}(1 \otimes 1)\right]\right| \\
& =\left|\operatorname{tr}\left[S_{z}(1 \otimes 1)-S U_{w}(1 \otimes 1) U_{w}\right]\right| \\
& =\left|\operatorname{tr}\left[S_{z}(1 \otimes 1)-S U_{z}\left(U_{z} U_{w} 1 \otimes U_{z} U_{w} 1\right) U_{z}\right]\right|
\end{aligned}
$$

From Lemma 2.8, the last term equals

$$
\begin{aligned}
\left|\operatorname{tr}\left[S_{z}\left(1 \otimes 1-U_{\phi_{w}(z)} 1 \otimes U_{\phi_{w}(z)} 1\right)\right]\right| & \leq\left\|S_{z}\right\|\left\|1 \otimes 1-U_{\phi_{w}(z)} 1 \otimes U_{\phi_{w}(z)} 1\right\|_{C^{1}} \\
& \leq \sqrt{2}\left\|S_{z}\right\|\left(2-2\left|\left\langle 1, k_{\phi_{w}(z)}\right\rangle\right|^{2}\right)^{1 / 2} \\
& =2\|S\|\left[1-\left(1-\left|\phi_{w}(z)\right|^{2}\right)^{n+1}\right]^{1 / 2} \\
& \leq C(n)\|S\|\left|\phi_{w}(z)\right|
\end{aligned}
$$

where the second equality holds by $\|T\|_{C^{1}} \leq \sqrt{l}\left(\operatorname{tr}\left[T^{*} T\right]\right)^{1 / 2}$ where $l$ is the rank of $T$. 
Suppose $\left|B_{m-1} S(z)-B_{m-1} S(w)\right|<C(m, n)\|S\| \rho(z, w)$. By Lemma 2.10, we have

$$
\begin{aligned}
& \left|B_{m} S(z)-B_{m} S(w)\right| \\
& \quad \leq \frac{m+n}{m}\left|B_{m-1} S(z)-B_{m-1} S(w)\right| \\
& \quad+\frac{m+n}{m} \sum_{i=1}^{n}\left|B_{m-1}\left(T_{\overline{\left(\phi_{z}\right)_{i}}} S T_{\left(\phi_{z}\right)_{i}}\right)(z)-B_{m-1}\left(T_{\overline{\left(\phi_{w}\right)_{i}}} S T_{\left(\phi_{w}\right)_{i}}\right)(w)\right| .
\end{aligned}
$$

Since the term in the summation is less than or equals

$$
\begin{aligned}
& \left|B_{m-1}\left(T_{\overline{\left(\phi_{z}\right)_{i}}} S T_{\left(\phi_{z}\right)_{i}}\right)(z)-B_{m-1}\left(T_{\overline{\left(\phi_{w}\right)_{i}}} S T_{\left(\phi_{z}\right)_{i}}\right)(z)\right| \\
& \quad+\left|B_{m-1}\left(T_{\overline{\left(\phi_{w}\right)_{i}}} S T_{\left(\phi_{z}\right)_{i}}\right)(z)-B_{m-1}\left(T_{\overline{\left(\phi_{w}\right)_{i}}} S T_{\left(\phi_{w}\right)_{i}}\right)(z)\right| \\
& \quad+\left|B_{m-1}\left(T_{\overline{\left(\phi_{w}\right)_{i}}} S T_{\left(\phi_{w}\right)_{i}}\right)(z)-B_{m-1}\left(T_{\overline{\left(\phi_{w}\right)_{i}}} S T_{\left(\phi_{w}\right)_{i}}\right)(w)\right|,
\end{aligned}
$$

it is sufficient to show that

$$
\left|B_{m-1}\left(T_{\overline{\left(\phi_{z}\right)_{i}}} S T_{\left(\phi_{z}\right)_{i}}\right)(z)-B_{m-1}\left(T_{\overline{\left(\phi_{w}\right)_{i}}} S T_{\left(\phi_{z}\right)_{i}}\right)(z)\right|<C(m, n)\|S\| \rho(z, w) .
$$

Lemma 2.8 gives

$$
\begin{aligned}
& \left|B_{m-1}\left(T \overline{\left(\phi_{z}\right)_{i}}-\overline{\left(\phi_{w}\right)_{i}} S T_{\left(\phi_{z}\right)_{i}}\right)(z)\right| \\
& =C_{n}^{m+n-1}\left|\operatorname{tr}\left[\left(T \overline{\left(\phi_{z}\right)_{i}}-\overline{\left(\phi_{w}\right)_{i}} S T_{\left(\phi_{z}\right)_{i}}\right) \sum_{z}^{m-1} C_{m-1, k} \frac{n ! k !}{(n+|k|) !} \frac{u^{k}}{\left\|u^{k}\right\|} \otimes \frac{u^{k}}{\left\|u^{k}\right\|}\right]\right| \\
& \leq C_{n}^{m+n-1} \sum_{|k|=0}^{m-1}\left|C_{m-1, k}\right| \frac{n ! k !}{(n+|k|) !}\left|\left\langle S_{z} T_{\left(\phi_{z}\right)_{i} \circ \phi_{z}} \frac{u^{k}}{\left\|u^{k}\right\|}, T_{\left(\left(\phi_{z}\right)_{i}-\left(\phi_{w}\right)_{i}\right) \circ \phi_{z}} \frac{u^{k}}{\left\|u^{k}\right\|}\right\rangle\right|
\end{aligned}
$$

$$
\leq C(m, n)\left\|S_{z}\right\|\left\|T_{\left(\left(\phi_{z}\right)_{i}-\left(\phi_{w}\right)_{i}\right) \circ \phi_{z}} \frac{u^{k}}{\left\|u^{k}\right\|}\right\|_{2} .
$$

Let $\lambda=\phi_{w}(z)$. Then

$$
\begin{aligned}
\left\|T_{\left(\left(\phi_{z}\right)_{i}-\left(\phi_{w}\right)_{i}\right) \phi_{z}} \frac{u^{k}}{\left\|u^{k}\right\|}\right\|_{2}^{2} & \leq \int_{B}\left|\left(\phi_{z} \circ \phi_{z}\right)_{i}(u)-\left(\phi_{w} \circ \phi_{z}\right)_{i}(u)\right|^{2} d u \\
& =\int_{B}\left|(\mathcal{U} u)_{i}-\left(\phi_{\lambda}(u)\right)_{i}\right|^{2} d u \\
& \leq 2 \int_{B}\left|(\mathcal{U} u)_{i}+u_{i}\right|^{2}+\left|u_{i}+\left(\phi_{\lambda}(u)\right)_{i}\right|^{2} d u
\end{aligned}
$$

where $\phi_{w} \circ \phi_{z}=\phi_{\lambda} \circ \mathcal{U}$ for some $\mathcal{U} \in \mathfrak{U}(n)$. 
Noting that

$$
\phi_{\lambda}(u)+u=\frac{\lambda-\langle u, \lambda\rangle u+\left[1-\left(1-|\lambda|^{2}\right)^{1 / 2}\right] Q_{\lambda}(u)}{1-\langle u, \lambda\rangle},
$$

we have that for $|\lambda| \leq 1 / 2$,

$$
\left|\phi_{\lambda}(u)+u\right| \leq 2\left(|\lambda|+|\lambda|+|\lambda|^{2}\right) \leq 6|\lambda| .
$$

By Lemma 2.8 we also have

$$
\int_{B}\left|(\mathcal{U} u)_{i}+u_{i}\right|^{2} d u=\int_{B}\left|((\mathcal{U}+I) u)_{i}\right|^{2} d u \leq C\|\mathcal{U}+I\|^{2} \leq C|\lambda|^{2} .
$$

Thus (2.6) is less than or equal to

$$
C(m, n)\left\|S_{z}\right\|\left[36|\lambda|^{2}+C|\lambda|^{2}\right]^{1 / 2} \leq C(m, n)\|S\||\lambda| .
$$

The proof is complete.

Lemma 2.12 Let $S \in \mathfrak{L}\left(L_{a}^{2}\right)$ and $m, j \geq 0$. If $\left|S^{*} K_{\lambda}(z)\right| \leq C$ for any $z \in B$ then $\left(B_{m} B_{j}\right)(S)=\left(B_{j} B_{m}\right)(S)$.

Proof. By Theorem 2.9, it is enough to show that $\left(B_{m} B_{j}\right) S(0)=$ $\left(B_{j} B_{m}\right) S(0)$. From Proposition 2.3, Proposition 2.1 and Fubini's Theorem, we have

$$
\begin{aligned}
B_{m}\left(B_{j} S\right)(0) & =B_{m}\left(T_{B_{j} S}\right)(0) \\
& =C_{n}^{m+n} \int_{B} B_{j} S(z)\left(1-|z|^{2}\right)^{m} d z \\
& =C_{n}^{m+n} C_{n}^{j+n} \int_{B} \int_{B} \int_{B}\left(1-|z|^{2}\right)^{m+j+n+1}(1-\langle u, \lambda\rangle)^{j} \times \\
& K_{z}^{j}(u) \overline{K_{z}^{j}(\lambda)} \overline{S^{*} K_{\lambda}(u)} d u d \lambda d z \\
= & C_{n}^{m+n} C_{n}^{j+n} \int_{B} \int_{B}(1-\langle u, \lambda\rangle)^{j} \int_{B}\left(1-|z|^{2}\right)^{m+j+n+1} \times \\
& K_{z}^{j}(u) \overline{K_{z}^{j}(\lambda)} d z \overline{S^{*} K_{\lambda}(u)} d u d \lambda .
\end{aligned}
$$

Let

$$
F_{m, j}(u, \lambda)=(1-\langle u, \lambda\rangle)^{j} \int_{B}\left(1-|z|^{2}\right)^{m+j+n+1} K_{z}^{j}(u) \overline{K_{z}^{j}(\lambda)} d z
$$

Then $F_{m, j}(u, \lambda)=\sum_{i=1}^{l} H_{i}(u) \overline{G_{i}}(\lambda)$ where $H_{i}$ and $G_{i}$ are holomorphic functions and for some $l \geq 0$. Thus, from Lemma 9 in [9], we just need to show $F_{m, j}(\lambda, \lambda)=F_{j, m}(\lambda, \lambda)$ for $\lambda \in B$. 
The change of variables implies

$$
\begin{aligned}
F_{m, j}(\lambda, \lambda) & =\left(1-|\lambda|^{2}\right)^{j} \int_{B}\left(1-|z|^{2}\right)^{m+j+n+1}\left|K_{\lambda}^{j}(z)\right|^{2} d z \\
& =\left(1-|\lambda|^{2}\right)^{j} \int_{B}\left(1-\left|\phi_{\lambda}(z)\right|^{2}\right)^{m+j+n+1}\left|K_{\lambda}^{j}\left(\phi_{\lambda}(z)\right)\right|^{2}\left|k_{\lambda}(z)\right|^{2} d z \\
& =\left(1-|\lambda|^{2}\right)^{m} \int_{B}\left(1-|z|^{2}\right)^{m+j+n+1}\left|K_{\lambda}^{m}(z)\right|^{2} d z \\
& =F_{j, m}(\lambda, \lambda)
\end{aligned}
$$

as desired.

Lemma 2.13 For any $S \in \mathfrak{L}\left(L_{a}^{2}\right)$, there exists sequences $\left\{S_{\alpha}\right\}$ satisfying

$$
\left|S_{\alpha}^{*} K_{\lambda}(u)\right| \leq C(\alpha)
$$

such that $B_{m}\left(S_{\alpha}\right)$ converges to $B_{m}(S)$ pointwise.

Proof. Since $H^{\infty}$ is dense in $L_{a}^{2}$ and the set of finite rank operators is dense in the ideal $\mathcal{K}$ of compact operators on $L^{2}$, the set $\left\{\sum_{i=1}^{l} f_{i} \otimes g_{i}\right.$ : $\left.f_{i}, g_{i} \in H^{\infty}\right\}$ is dense in the ideal $\mathcal{K}$ in the norm topology. Since $\mathcal{K}$ is dense in the space of bounded operators on $L_{a}^{2}$ in strong operator topology, (2.3) gives that for any $S \in \mathfrak{L}\left(L_{a}^{2}\right)$, there exists a finite rank operator sequences $S_{\alpha}=\sum_{i=1}^{l} f_{i} \otimes g_{i}$ such that $B_{m}\left(S_{\alpha}\right)$ converges to $B_{m}(S)$ pointwise for some $f_{i}, g_{i}$ in $H^{\infty}$. Also, for $l \geq 0$, for such $S_{\alpha}=\sum_{i=1}^{l} f_{i} \otimes g_{i}$, we have

$$
\begin{aligned}
\left|S_{\alpha}^{*} K_{\lambda}(u)\right| & =\left|\sum_{i=1}^{l}\left(g_{i} \otimes f_{i}\right) K_{\lambda}(u)\right|=\left|\sum_{i=1}^{l}\left\langle K_{\lambda}(u), f_{i}(u)\right\rangle g_{i}(u)\right| \\
& \leq \sum_{i=1}^{l}\left|f_{i}(\lambda)\right|\left|g_{i}(u)\right| \leq \sum_{i=1}^{l}\left\|f_{i}\right\|_{\infty}\left\|g_{i}\right\|_{\infty}<C .
\end{aligned}
$$

The proof is complete.

Proposition 2.14 Let $S \in \mathfrak{L}\left(L_{a}^{2}\right)$ and $m, j \geq 0$. Then

$$
\left(B_{m} B_{j}\right)(S)=\left(B_{j} B_{m}\right)(S) \text {. }
$$

Proof. Let $S \in \mathfrak{L}\left(L_{a}^{2}\right)$. Then Lemma 2.13 implies that there exists a sequence $\left\{S_{\alpha}\right\}$ satisfying $\left|S_{\alpha}^{*} K_{\lambda}(u)\right| \leq C(\alpha)$, hence $B_{m}\left(B_{j} S_{\alpha}\right)(z)=B_{j}\left(B_{m} S_{\alpha}\right)(z)$ by Lemma 2.12. From Proposition 2.3, we know

$$
B_{m}\left(B_{j} S_{\alpha}\right)(z)=\int_{B}\left(B_{j} S_{\alpha}\right) \circ \phi_{z}(u) d \nu_{m}(u)
$$

and $\left\|\left(B_{j} S_{\alpha}\right) \circ \phi_{z}\right\|_{\infty} \leq C(j, n)\|S\|$. Also, $\left(B_{j} S_{\alpha}\right) \circ \phi_{z}(u)$ converges to $\left(B_{j} S\right) \circ$ $\phi_{z}(u)$. Therefore $B_{m}\left(B_{j} S_{\alpha}\right)(z)$ converges to $B_{m}\left(B_{j} S\right)(z)$. By the uniqueness of the limit, we have $\left(B_{m} B_{j}\right)(S)=\left(B_{j} B_{m}\right)(S)$. 
Proposition 2.15 Let $S \in \mathfrak{L}\left(L_{a}^{2}\right)$ and $m \geq 0$. If $B_{0} S(z) \rightarrow 0$ as $z \rightarrow \partial B$ then $B_{m} S(z) \rightarrow 0$ as $z \rightarrow \partial B$.

Proof. Suppose $B_{0} S(z) \rightarrow 0$ as $z \rightarrow \partial B$. Then we will prove that $S_{z} \rightarrow 0$ in the $\mathcal{T}^{*}$-norm as $z \rightarrow \partial B$. Suppose it is not true. Then for some net $\left\{w_{\alpha}\right\} \in B$ and an operator $V \neq 0$ in $\mathfrak{L}\left(L_{a}^{2}\right)$, there exists a sequence $\left\{S_{w_{\alpha}}\right\}$ such that $S_{w_{\alpha}} \rightarrow V$ in the $\mathcal{T}^{*}$-norm as $w_{\alpha} \rightarrow \partial B$, hence $\operatorname{tr}\left[S_{w_{\alpha}} T\right] \rightarrow \operatorname{tr}[V T]$ for any $T \in \mathcal{T}$. Let $T=k_{\lambda} \otimes k_{\lambda}$ for fixed $\lambda \in B$. Then Theorem 2.9 implies

$$
\begin{aligned}
\operatorname{tr}\left[S_{w_{\alpha}} T\right] & =\operatorname{tr}\left[S_{w_{\alpha}}\left(k_{\lambda} \otimes k_{\lambda}\right)\right]=\left\langle S_{w_{\alpha}} k_{\lambda}, k_{\lambda}\right\rangle \\
& =B_{0} S_{w_{\alpha}}(\lambda)=\left(B_{0} S\right) \circ \phi_{w_{\alpha}}(\lambda) \rightarrow 0
\end{aligned}
$$

as $w_{\alpha} \rightarrow \partial B$. Since $\operatorname{tr}[V T]=B_{0} V(\lambda)$ and $B_{0}$ is one-to-one mapping, $V=0$. This is the contradiction. Thus $S_{z} \rightarrow 0$ as $z \rightarrow \partial B$ in the $\mathcal{T}^{*}$-norm. (1.2) finishes the proof of this proposition.

\section{Operators $S$ approximated by Toeplitz operators $T_{B_{m}(S)}$}

In this section we will give a criterion for operators approximated by Toeplitz operators with symbol equal to their $m$-Berezin transforms. The main result in this section is Theorem 3.7. It extends and improves Theorem 2.4 in [17]. Even on the unit disk, we will show an example that the result in the theorem is sharp on the unit disk.

From Proposition 1.4.10 in [14], we have the following lemma

Lemma 3.1 Suppose $a<1$ and $a+b<n+1$. Then

$$
\sup _{z \in B} \int_{B} \frac{d \lambda}{\left(1-|\lambda|^{2}\right)^{a}|1-\langle\lambda, z\rangle|^{b}}<\infty .
$$

This lemma gives the following lemma which extends Lemma 4.2 in [13].

Let $1<q<\infty$ and $p$ be the conjugate exponent of $q$. If we take $p>n+2$, then $q<(n+2) /(n+1)$.

Lemma 3.2 Let $S \in \mathfrak{L}\left(L_{a}^{2}\right)$ and $p>n+2$. Then there exists $C(n, p)>0$ such that $h(z)=\left(1-|z|^{2}\right)^{-a}$ where $a=(n+1) /(n+2)$ satisfies

$$
\int_{B}\left|\left(S K_{z}\right)(w)\right| h(w) d w \leq C(n, p)\left\|S_{z} 1\right\|_{p} h(z)
$$

for all $z \in B$ and

$$
\int_{B}\left|\left(S K_{z}\right)(w)\right| h(z) d z \leq C(n, p)\left\|S_{w}^{*} 1\right\|_{p} h(w)
$$

for all $w \in B$. 
Proof. Fix $z \in B$. Since

$$
U_{z} 1=(-1)^{n}\left(1-|z|^{2}\right)^{(n+1) / 2} K_{z},
$$

we have

$$
\begin{aligned}
S K_{z} & =(-1)^{n}\left(1-|z|^{2}\right)^{-(n+1) / 2} S U_{z} 1 \\
& =(-1)^{n}\left(1-|z|^{2}\right)^{-(n+1) / 2} U_{z} S_{z} 1 \\
& =\left(1-|z|^{2}\right)^{-(n+1) / 2}\left(S_{z} 1 \circ \phi_{z}\right) k_{z} .
\end{aligned}
$$

Thus, letting $\lambda=\phi_{z}(w)$, the change of variables implies

$$
\begin{aligned}
\int_{B} \frac{\left|\left(S K_{z}\right)(w)\right|}{\left(1-|w|^{2}\right)^{a}} d w & =\frac{1}{\left(1-|z|^{2}\right)^{(n+1) / 2}} \int_{B} \frac{\left|\left(S_{z} 1 \circ \phi_{z}\right)(w)\right|\left|k_{z}(w)\right|}{\left(1-|w|^{2}\right)^{a}} d w \\
& =\frac{1}{\left(1-|z|^{2}\right)^{a}} \int_{B} \frac{\left|S_{z} 1(\lambda)\right|}{\left(1-|\lambda|^{2}\right)^{a}|1-\langle\lambda, z\rangle|^{n+1-2 a}} d \lambda \\
& \leq \frac{1 \mid S_{z} 1 \|_{p}}{\left(1-|z|^{2}\right)^{a}}\left(\int_{B} \frac{1}{\left(1-|\lambda|^{2}\right)^{a q}|1-\langle\lambda, z\rangle|^{(n+1-2 a) q}} d \lambda\right)^{\frac{1}{q}}
\end{aligned}
$$

The last inequality comes from Holder's inequality. Since $a q<1$ and $a q+$ $(n+1-2 a) q<n+1$, Lemma 3.1 implies (3.1).

To prove (3.2), replace $S$ by $S^{*}$ in (3.1), interchange $w$ and $z$ in (3.1) and then use the equation

$$
\left(S^{*} K_{w}\right)(z)=\left\langle S^{*} K_{w}, K_{z}\right\rangle=\left\langle K_{w}, S K_{z}\right\rangle=\overline{S K_{z}}(w)
$$

to obtain the desired result.

Lemma 3.3 Let $S \in \mathfrak{L}\left(L_{a}^{2}\right)$ and $p>n+2$. Then

$$
\|S\| \leq C(n, p)\left(\sup _{z \in B}\left\|S_{z} 1\right\|_{p}\right)^{1 / 2}\left(\sup _{z \in B}\left\|S_{z}^{*} 1\right\|_{p}\right)^{1 / 2}
$$

where $C(n, p)$ is the constant of Lemma 3.2.

Proof. (3.3) implies

$$
(S f)(w)=\left\langle S f, K_{w}\right\rangle=\int_{B} f(z) \overline{\left(S^{*} K_{w}\right)}(z) d z=\int_{B} f(z)\left(S K_{z}\right)(w) d z
$$

for $f \in L_{a}^{2}$ and $w \in B$. Thus, Lemma 3.2 and the classical Schur's theorem finish the proof. 
884 K. NAm, D. Zheng And C. Zhong

Lemma 3.4 Let $S_{m}$ be a bounded sequence in $\mathfrak{L}\left(L_{a}^{2}\right)$ such that

$$
\left\|B_{0} S_{m}\right\|_{\infty} \rightarrow 0 \quad \text { as } m \rightarrow \infty \text {. }
$$

Then

$$
\sup _{z \in B}\left|\left\langle\left(S_{m}\right)_{z} 1, f\right\rangle\right| \rightarrow 0
$$

as $m \rightarrow \infty$ for any $f \in L_{a}^{2}$ and

$$
\sup _{z \in B}\left|\left(S_{m}\right)_{z} 1\right| \rightarrow 0
$$

uniformly on compact subsets of $B$ as $m \rightarrow \infty$.

Proof. To prove (3.4), we only need to have

$$
\sup _{z \in B}\left|\left\langle\left(S_{m}\right)_{z} 1, w^{k}\right\rangle\right| \rightarrow 0
$$

as $m \rightarrow \infty$ for any multi-index $k$.

Since

$$
K_{z}(w)=\sum_{|\alpha|=0}^{\infty} \frac{(n+|\alpha|) !}{n ! \alpha !} \bar{z}^{\alpha} w^{\alpha}
$$

we have

$$
\begin{aligned}
& B_{0} S_{m}\left(\phi_{z}(\lambda)\right)=B_{0}\left(S_{m}\right)_{z}(\lambda) \\
& =\left(1-|\lambda|^{2}\right)^{n+1} \sum_{|\alpha|=0}^{\infty} \sum_{|\beta|=0}^{\infty} \frac{(n+|\alpha|) !}{n ! \alpha !} \frac{(n+|\beta|) !}{n ! \beta !}\left\langle\left(S_{m}\right)_{z} w^{\alpha}, w^{\beta}\right\rangle \bar{\lambda}^{\alpha} \lambda^{\beta}
\end{aligned}
$$

where $\alpha, \beta$ are multi-indices.

Then for any fixed $k$ and $0<r<1$,

$$
\begin{aligned}
\int_{r B} & \frac{B_{0} S_{m}\left(\phi_{z}(\lambda)\right) \bar{\lambda}^{k}}{\left(1-|\lambda|^{2}\right)^{n+1}} d \lambda \\
= & \sum_{|\alpha|=0}^{\infty} \sum_{|\beta|=0}^{\infty} \frac{(n+|\alpha|) !}{n ! \alpha !} \frac{(n+|\beta|) !}{n ! \beta !}\left\langle\left(S_{m}\right)_{z} w^{\alpha}, w^{\beta}\right\rangle \int_{r B} \bar{\lambda}^{\alpha+k} \lambda^{\beta} d \lambda \\
= & r^{2 n+2|k|}\left(\left\langle\left(S_{m}\right)_{z} 1, w^{k}\right\rangle+\sum_{|\alpha|=1}^{\infty} \frac{(n+|\alpha|) !}{n ! \alpha !}\left\langle\left(S_{m}\right)_{z} w^{\alpha}, w^{\alpha+k}\right\rangle r^{2|\alpha|}\right)
\end{aligned}
$$


Since $S_{m}$ is bounded sequence, we have

$$
\begin{aligned}
\left|\left\langle\left(S_{m}\right)_{z} 1, w^{k}\right\rangle\right| \leq r^{-2 n-2|k|}\left|\int_{r B} \frac{B_{0} S_{m}\left(\phi_{z}(\lambda)\right) \bar{\lambda}^{k}}{\left(1-|\lambda|^{2}\right)^{n+1}} d \lambda\right|+ \\
\qquad \sum_{|\alpha|=1}^{\infty} \frac{(n+|\alpha|) !}{n ! \alpha !}\left\|\left(S_{m}\right)_{z}\right\|\left\|w^{\alpha}\right\|\left\|w^{\alpha+k}\right\| r^{2|\alpha|} \\
\leq r^{-2 n-2|k|}\left\|B_{0} S_{m}\right\|_{\infty} \int_{r B} \frac{\left|\lambda^{k}\right|}{\left(1-|\lambda|^{2}\right)^{n+1}} d \lambda+C \sum_{|\alpha|=1}^{\infty} r^{2|\alpha|}
\end{aligned}
$$

hence, by assumption

$$
\limsup _{m \rightarrow \infty} \sup _{z \in B}\left|\left\langle\left(S_{m}\right)_{z} 1, w^{k}\right\rangle\right| \leq C \sum_{|\alpha|=1}^{\infty} r^{2|\alpha|} .
$$

Letting $r \rightarrow 0$, we have (3.6).

Now we prove (3.5). From (3.7), we get

$$
\begin{aligned}
\left|\left(S_{m}\right)_{z} 1(\lambda)\right| & =\left|\left\langle\left(S_{m}\right)_{z} 1, K_{\lambda}\right\rangle\right| \\
& \leq \sum_{|\alpha|=0}^{\infty} \frac{(n+|\alpha|) !}{n ! \alpha !}\left|\left\langle\left(S_{m}\right)_{z} 1, w^{\alpha}\right\rangle\right|\left|\lambda^{\alpha}\right| \\
& \leq \sum_{|\alpha|=0}^{l-1} \frac{(n+|\alpha|) !}{n ! \alpha !}\left|\left\langle\left(S_{m}\right)_{z} 1, w^{\alpha}\right\rangle\right|+\sum_{|\alpha|=l}^{\infty} \frac{(n+|\alpha|) !}{n ! \alpha !}\left\|S_{m}\right\|\left\|w^{\alpha}\right\|\left|\lambda^{\alpha}\right|
\end{aligned}
$$

for $z \in B, \lambda \in r B$ and $l \geq 1$. Since the second summation is less than or equals to

$$
\begin{aligned}
\sum_{j=l}^{\infty}\left(\frac{(n+j) !}{n ! j !}\right)^{1 / 2} \sum_{|\alpha|=j}\left(\frac{j !}{\alpha !}\right)^{1 / 2}\left|\lambda^{\alpha}\right| & \leq \sum_{j=l}^{\infty} \frac{(n+j) !}{n ! j !}\left[\sum_{|\alpha|=j} \frac{j !}{\alpha !}\left|\lambda^{\alpha}\right|^{2}\right]^{1 / 2} \\
& \leq \sum_{j=l}^{\infty} \frac{(n+j) !}{n ! j !} r^{j}
\end{aligned}
$$

for any $\epsilon>0$, we can find sufficiently large $l$ such that the second summation is less than $\epsilon$. Thus, (3.6) imply $\sup _{z \in B}\left|\left(S_{m}\right)_{z} 1\right| \rightarrow 0$ uniformly on compact subsets of $B$ as $m \rightarrow \infty$.

Lemma 3.5 Let $\left\{S_{m}\right\}$ be a sequence in $\mathfrak{L}\left(L_{a}^{2}\right)$ such that for some $p>n+2$, $\left\|B_{0} S_{m}\right\|_{\infty} \rightarrow 0$ as $m \rightarrow \infty$,

$$
\sup _{z \in B}\left\|\left(S_{m}\right)_{z} 1\right\|_{p} \leq C \quad \text { and } \quad \sup _{z \in B}\left\|\left(S_{m}^{*}\right)_{z} 1\right\|_{p} \leq C
$$

where $C>0$ is independent of $m$, then $S_{m} \rightarrow 0$ as $m \rightarrow \infty$ in $\mathfrak{L}\left(L_{a}^{2}\right)$-norm. 
Proof. Lemma 3.3 implies

$$
\left\|S_{m}\right\| \leq C(n, p)\left(\sup _{z \in B}\left\|\left(S_{m}\right)_{z} 1\right\|_{p}\right)^{1 / 2}\left(\sup _{z \in B}\left\|\left(S_{m}^{*}\right)_{z} 1\right\|_{p}\right)^{1 / 2} \leq C(n, p),
$$

hence, Lemma 3.4 gives

$$
\sup _{z \in B}\left|\left(S_{m}\right)_{z} 1\right| \rightarrow 0
$$

uniformly on compact subsets of $B$ as $m \rightarrow \infty$.

Here, for $n+2<s<p$, Holder's inequality gives

$$
\begin{aligned}
\sup _{z \in B}\left\|\left(S_{m}\right)_{z} 1\right\|_{s}^{s} & \leq \sup _{z \in B} \int_{B \backslash r \bar{B}}\left|\left(S_{m}\right)_{z} 1(w)\right|^{s} d w+\sup _{z \in B} \int_{r \bar{B}}\left|\left(S_{m}\right)_{z} 1(w)\right|^{s} d w \\
& \leq C \sup _{z \in B}\left\|\left(S_{m}\right)_{z} 1\right\|_{p}^{s}(1-r)^{1-s / p}+\sup _{z \in B} \int_{r \bar{B}}\left|\left(S_{m}\right)_{z} 1(w)\right|^{s} d w
\end{aligned}
$$

and (3.8) implies the second term tends to 0 as $m \rightarrow \infty$. Also, the first term is less than or equals to $C^{s}(1-r)^{1-s / p}$ which can be small by taking $r$ close to 1 . Consequently, Lemma 3.3 gives

$$
\begin{aligned}
\left\|S_{m}\right\| & \leq C(n, s)\left(\sup _{z \in B}\left\|\left(S_{m}\right)_{z} 1\right\|_{s}\right)^{1 / 2}\left(\sup _{z \in B}\left\|\left(S_{m}^{*}\right)_{z} 1\right\|_{s}\right)^{1 / 2} . \\
& \leq C(n, s)\left(\sup _{z \in B}\left\|\left(S_{m}\right)_{z} 1\right\|_{s}\right)^{1 / 2} \rightarrow 0
\end{aligned}
$$

Corollary 3.6 Let $S \in \mathfrak{L}\left(L_{a}^{2}\right)$ such that for some $p>n+2$,

$$
\sup _{z \in B}\left\|S_{z} 1-\left(T_{B_{m} S}\right)_{z} 1\right\|_{p} \leq C \quad \text { and } \quad \sup _{z \in B}\left\|S_{z}^{*} 1-\left(T_{B_{m}\left(S^{*}\right)}\right)_{z} 1\right\|_{p} \leq C,
$$

where $C>0$ is independent of $m$. Then $T_{B_{m} S} \rightarrow S$ as $m \rightarrow \infty$ in $\mathfrak{L}\left(L_{a}^{2}\right)$ norm.

Proof. Let $S_{m}=S-T_{B_{m} S}$. Then Proposition 2.14 and Theorem 2.11 imply

$$
B_{0}\left(S_{m}\right)=B_{0} S-B_{0}\left(T_{B_{m} S}\right)=B_{0} S-B_{0}\left(B_{m} S\right)=B_{0} S-B_{m}\left(B_{0} S\right)
$$

which tends uniformly to 0 as $m \rightarrow \infty$, hence $\left\|B_{0}\left(S_{m}\right)\right\|_{\infty} \rightarrow 0$. Consequently, by Lemma 3.5 we complete the proof. 
Theorem 3.7 Let $S \in \mathfrak{L}\left(L_{a}^{2}\right)$. If there is $p>n+2$ such that

$$
\sup _{z \in B}\left\|T_{\left(B_{m} S\right) \circ \phi_{z}} 1\right\|_{p}<C \quad \text { and } \quad \sup _{z \in B}\left\|T_{\left(B_{m} S\right) \circ \phi_{z}}^{*} 1\right\|_{p}<C
$$

where $C>0$ is independent of $m$, then $T_{B_{m} S} \rightarrow S$ as $m \rightarrow \infty$ in $\mathfrak{L}\left(L_{a}^{2}\right)$ norm.

Proof. By Corollary 3.6, we only need to show that (3.10) implies (3.9). Since $T_{\left(B_{m} S\right)_{\circ} \phi_{z}}=\left(T_{B_{m} S}\right)_{z}$ and

$$
T_{\left(B_{m} S\right) \circ \phi_{z}}^{*}=T_{\overline{B_{m} S_{z}}}=T_{B_{m}\left(S_{z}^{*}\right)}=T_{\left(B_{m}\left(S^{*}\right)\right) \circ \phi_{z}},
$$

it is sufficient to show that

$$
\sup _{z \in B}\left\|S_{z} 1\right\|_{p}<\infty
$$

By Lemma 3.3, we get

$$
\left\|T_{B_{m} S}\right\| \leq C(n, p)\left(\sup _{z \in B}\left\|T_{B_{m} S \circ \phi_{z}} 1\right\|_{p}\right)^{1 / 2}\left(\sup _{z \in B}\left\|T_{B_{m} S \circ \phi_{z}}^{*} 1\right\|_{p}\right)^{1 / 2}<C
$$

where $C$ is independent of $m$, hence writing $S_{m}=S-T_{B_{m} S}$, we have $\left\|S_{m}\right\| \leq C$ where $C$ is independent of $m$. Also, the proof of Corollary 3.6 implies

$$
\left\|B_{0} S_{m}\right\|_{\infty} \rightarrow 0
$$

as $m \rightarrow \infty$.

Let $f$ be a polynomial with $\|f\|_{q}=1$. Then Lemma 3.4 implies

$$
\sup _{z \in B}\left|\left\langle\left(S_{m}\right)_{z} 1, f\right\rangle\right| \rightarrow 0
$$

as $m \rightarrow \infty$. Thus, for any $\epsilon>0$ and $z_{0} \in B$, we have

$$
\left|\left\langle S_{z_{0}} 1, f\right\rangle\right| \leq \sup _{z \in B}\left|\left\langle\left(S_{m}\right)_{z} 1, f\right\rangle\right|+\left|\left\langle\left(T_{B_{m} S}\right)_{z_{0}} 1, f\right\rangle\right| \leq \epsilon+C
$$

for sufficiently large $m$, where $C$ is independent of $m$. Since $\epsilon$ is arbitrary, we get

$$
\sup _{z \in B}\left\|S_{z} 1\right\|_{p}<\infty
$$

as desired. 


\section{Compact Radial operator}

Given $\mathcal{U} \in \mathfrak{U}(n)$, define $V_{\mathcal{U}} f(w)=f(\mathcal{U} w) \operatorname{det} \mathcal{U}$ for $f \in L_{a}^{2}$. Then $V_{\mathcal{U}}$ is a unitary operator on $L_{a}^{2}$. We say that $S \in \mathfrak{L}\left(L_{a}^{2}\right)$ is a radial operator if $S V_{\mathcal{U}}=V_{\mathcal{U}} S$ for any $\mathcal{U} \in \mathfrak{U}(n)$.

If $S \in \mathfrak{L}\left(L_{a}^{2}\right)$, the radialization of $S$ is defined by

$$
S^{\sharp}=\int_{\mathfrak{U}} V_{\mathcal{U}}^{*} S V_{\mathcal{U}} d \mathcal{U}
$$

where $d \mathcal{U}$ is the Haar measure on the compact group $\mathfrak{U}(n)$ and the integral is taken in the weak sense. Then $S^{\sharp}=S$ if $S$ is radial and $\mathfrak{U}$-invariance of $d \mathcal{U}$ shows that $S^{\sharp}$ is indeed a radial operator.

If $f \in L^{\infty}$ and $g, h \in L_{a}^{2}$ then

$$
\left\langle V_{\mathcal{U}}{ }^{*} T_{f} V_{\mathcal{U}} g, h\right\rangle=\int_{B} f(w) V_{\mathcal{U}} g(w) \overline{V_{\mathcal{U}} h(w)} d w=\int_{B} f\left(\mathcal{U}^{*} w\right) g(w) \overline{h(w)} d w .
$$

Thus $V_{\mathcal{U}}{ }^{*} T_{f} V_{\mathcal{U}}=T_{f \circ \mathcal{U}^{*}}$ and

$$
V_{\mathcal{U}}{ }^{*} T_{f_{1}} \cdots T_{f_{l}} V_{\mathcal{U}}=T_{f_{1} \circ \mathcal{U}^{*}} \cdots T_{f_{l} \circ \mathcal{U}^{*}}
$$

for $f_{1}, \ldots, f_{l} \in L^{\infty}, l \geq 0$.

Lemma 4.1 Let $S \in \mathfrak{L}\left(L_{a}^{2}\right)$ be a radial operator. Then $T_{B_{m}(S)}=\int_{B} S_{w} d \nu_{m}(w)$.

Proof. Let $z \in B$. By (2.3) and Lemma 2.8, we obtain

$$
\begin{aligned}
B_{0}\left(\int_{B} S_{w} d \nu_{m}(w)\right)(z) & =\left\langle\left(\int_{B} S_{w} d \nu_{m}(w)\right)_{z} 1,1\right\rangle \\
& =\int_{B}\left\langle U_{z} U_{w} S U_{w} U_{z} 1,1\right\rangle d \nu_{m}(w) \\
& =\int_{B}\left\langle U_{\phi_{z}(w)} V_{\mathcal{U}}^{*} S V_{\mathcal{U}} U_{\phi_{z}(w)} 1,1\right\rangle d \nu_{m}(w)
\end{aligned}
$$

where $V_{\mathcal{U}}$ is in Lemma 2.8. Since $S$ is a radial operator, Theorem 2.9, Proposition 2.3 and Proposition 2.14 imply that the last integral equals

$$
\begin{aligned}
\int_{B}\left\langle U_{\phi_{z}(w)} S U_{\phi_{z}(w)} 1,1\right\rangle d \nu_{m}(w) & =\int_{B} B_{0} S \circ \phi_{z}(w) d \nu_{m}(w) \\
& =B_{m} B_{0} S(z) \\
& =B_{0} B_{m} S(z) \\
& =B_{0}\left(T_{B_{m}(S)}\right)(z)
\end{aligned}
$$

Since $B_{0}$ is one-to-one mapping, the proof is complete. 
Theorem 4.2 Let $S \in \mathfrak{T}\left(L^{\infty}\right)$ be a radial operator. Then $S$ is compact if and only if $B_{0} S \equiv 0$ on $\partial B$.

Proof. Suppose $B_{0} S \equiv 0$ on $\partial B$. Then $B_{m} S \equiv 0$ on $\partial B$ by Proposition 2.15, hence $T_{B_{m} S}$ is compact for all $m \geq 0$.

Let

$$
Q=\int_{\mathfrak{U}} T_{f_{1} \circ \mathcal{U}^{*}} \cdots T_{f_{l} \circ \mathcal{U}^{*}} d \mathcal{U}
$$

with $f_{1}, \ldots, f_{l} \in L^{\infty}$ for some $l \geq 0$. Then $Q \in \mathfrak{L}\left(L_{a}^{2}\right)$. By Lemma 4.1, for any $z \in B$, we have

$$
\begin{aligned}
T_{\left(B_{m}(Q)\right) \circ \phi_{z}} & =\int_{B}\left((Q)_{z}\right)_{w} d \nu_{m}(w) \\
& =\int_{B} \int_{\mathfrak{U}} T_{f_{1} \circ \mathcal{U}^{*} \circ \phi_{z} \circ \phi_{w}} \cdots T_{f_{l} \circ \mathcal{U}^{*} \circ \phi_{z} \circ \phi_{w}} d \mathcal{U} d \nu_{m}(w) .
\end{aligned}
$$

Consequently,

$$
\begin{aligned}
\left\|T_{\left(B_{m}(Q)\right) \circ \phi_{z}}\right\| & \leq C(l)\left\|f_{1} \circ \mathcal{U}^{*} \circ \phi_{z} \circ \phi_{w}\right\|_{\infty} \cdots\left\|f_{l} \circ \mathcal{U}^{*} \circ \phi_{z} \circ \phi_{w}\right\|_{\infty} \\
& =C(l)\left\|f_{1}\right\|_{\infty} \cdots\left\|f_{l}\right\|_{\infty} .
\end{aligned}
$$

Similarly, we have

$$
\left\|T_{\left(B_{m}(Q)\right) \circ \phi_{z}}^{*}\right\| \leq C(l)\left\|f_{1}\right\|_{\infty} \cdots\left\|f_{l}\right\|_{\infty} .
$$

Thus, Theorem 3.7 gives that

$$
T_{B_{m}(Q)} \rightarrow Q
$$

in $\mathfrak{L}\left(L_{a}^{2}\right)$-norm.

Since $S \in \mathfrak{T}\left(L^{\infty}\right)$, there exists a sequence $\left\{S_{k}\right\}$ such that $S_{k} \rightarrow S$ in $\mathfrak{L}\left(L_{a}^{2}\right)$-norm where each $S_{k}$ is a finite sum of finite products of Toeplitz operators. Since the radialization is continuous and $S$ is radial, $S_{k}^{\sharp} \rightarrow S^{\sharp}=S$. From Lemma 4.1, we have

$$
\left\|T_{B_{m} S}\right\|=\left\|\int_{B} S_{w} d \nu_{m}(w)\right\| \leq \int_{B}\left\|S_{w}\right\| d \nu_{m}(w)=\|S\|
$$

Thus

$$
\begin{aligned}
\left\|S-T_{B_{m} S}\right\| & \leq\left\|S-S_{k}^{\sharp}\right\|+\left\|S_{k}^{\sharp}-T_{B_{m}\left(S_{k}^{\sharp}\right)}\right\|+\left\|T_{B_{m}\left(S_{k}^{\sharp}\right)}-T_{B_{m} S}\right\| \\
& \leq 2\left\|S-S_{k}^{\sharp}\right\|+\left\|S_{k}^{\sharp}-T_{B_{m}\left(S_{k}^{\sharp}\right)}\right\|
\end{aligned}
$$

and (4.1) imply $T_{B_{m}(S)} \rightarrow S$ as $m \rightarrow \infty$ in $\mathfrak{L}\left(L_{a}^{2}\right)$-norm, hence $S$ is compact. The other direction is trivial. 
Example. This example shows that for $n=1$, the number $n+2=3$ in Theorem 3.7 is sharp. We show that there is a bounded operator $S$ on $L_{a}^{2}$ such that

$$
\sup _{z \in D} \max \left\{\left\|T_{\left(B_{m} S\right) \circ \phi_{z}} 1\right\|_{3},\left\|T_{\left(B_{m} S\right) \circ \phi_{z}}^{*} 1\right\|_{3}\right\}<\infty,
$$

and for each $m \geq 0, B_{m}(S)(z) \rightarrow 0$ as $z \rightarrow \partial D$, but $S$ is not compact on $L_{a}^{2}$.

The following operator $S$ was constructed in [3] to show that $B_{0}(S)(z) \rightarrow$ 0 as $z \rightarrow \partial D$, but $S$ is not compact on $L_{a}^{2}$. Let $S$ be defined on $L_{a}^{2}$ by

$$
S\left(\sum_{l=0}^{\infty} a_{l} w^{l}\right)=\sum_{l=0}^{\infty} a_{2^{l}} w^{2^{l}}
$$

It is clear that $S$ is a self-adjoint projection with infinite-dimensional range. Thus $S$ is not compact on $L_{a}^{2}$. From

$$
B_{0}(S)(z)=\left\langle S k_{z}, k_{z}\right\rangle=\left\|S k_{z}\right\|_{2}^{2}=\left(1-|z|^{2}\right)^{2} \sum_{l=0}^{\infty}\left(2^{l}+1\right)\left(|z|^{2}\right)^{2^{l}}
$$

it is easy to see that

$$
B_{0}(S)(z) \rightarrow 0 \quad \text { as } z \rightarrow \partial D
$$

By Proposition 2.15, we see that

$$
B_{m}(S)(z) \rightarrow 0 \quad \text { as } z \rightarrow \partial D .
$$

This gives that $T_{B_{m}(S)}$ is compact. Hence $T_{B_{m}(S)}$ does not converge to $S$ in the norm topology.

By means of the Zygmund theorem on gap series [18], it was proved in [13] that

$$
C=\sup _{z \in D} \max \left\{\left\|S_{z} 1\right\|_{3},\left\|S_{z}^{*} 1\right\|_{3}\right\}<\infty .
$$

Clearly, $S$ is a radial operator. By Lemma 4.1, we have

$$
T_{\left(B_{m} S\right) \circ \phi_{z}} 1=\int_{D}\left(S_{w}\right)_{z} 1 d \nu_{m}(w)=\int_{D} S_{\phi_{z}(w)} 1 d \nu_{m}(w)=\int_{D} S_{\lambda} 1 d \nu_{m} \circ \phi_{z}(\lambda) .
$$

Noting that for each $z \in D, d \nu_{m} \circ \phi_{z}$ is a probability measure on $D$, we have

$$
\left\|T_{\left(B_{m} S\right) \circ \phi_{z}} 1\right\|_{3} \leq \int_{D}\left\|S_{\lambda} 1\right\|_{3} d \nu_{m} \circ \phi_{z}(\lambda) \leq C .
$$

Similarly, we also have

$$
\left\|T_{\left(B_{m} S\right) \circ \phi_{z}}^{*} 1\right\|_{3} \leq C
$$




\section{References}

[1] Ahern, P., Flores, M. And Rudin, W.: An invariant volume-meanvalue property. J. Funct. Anal. 111 (1993), 380-397.

[2] Axler, S. And Zheng, D.: The Berezin transform on the Toeplitz algebra. Studia Math. 127 (1998), 113-136.

[3] Axler, S. And Zheng, D.: Compact operators via the Berezin transform. Indiana Univ. Math. J. 47 (1998), 387-399.

[4] Békollé, D., Berger, C. A., Coburn, L. A. And Zhu, K. H.: BMO in the Bergman metric on bounded symmetric domains. J. Funct. Anal. 93 (1990), 310-350.

[5] Berezin, F. A.: Covariant and contravariant symbols of operators. Math. USSR-IZv. 6 (1972), 1117-1151.

[6] Berger, C. A. and Coburn, L. A.: Toeplitz operators on the SegalBargmann space. Trans. Amer. Math. Soc. 301 (1987), 813-829.

[7] Berger, C. A. and Coburn, L. A.: Heat flow and Berezin-Toeplitz estimates. Amer. J. Math. 116 (1994), 563-590.

[8] Bratteli, O. and Robinson, D. W.: Operator algebras and quantum statistical mechanics. I, II. Texts and Monographs in Physics. Springer Verlag, New York 1979, 1981.

[9] Choe, B.R. And Lee, Y.J.: Pluriharmonic symbols of commuting Toeplitz operators. Illinois J. Math. 37 (1993), 424-436.

[10] Coburn, L. A.: A Lipschitz estimate for Berezin's operator calculus. Proc. Amer. Math. Soc. 133 (2005), 127-131.

[11] Engliš, M.: Compact Toeplitz operators via the Berezin transform on bounded symmetric domains. Integral Equations Operator Theory $\mathbf{3 3}$ (1999), 426-455.

[12] Gohberg, I. C. And KreIn, M. G.: Introduction to the theory of linear nonselfadjoint operators. Translations of Mathematical Monographs 18. American Mathematical Society, Providence, R.I., 1969.

[13] Miao, J. And Zheng, D.: Compact operators on Bergman spaces. Integral Equations Operator Theory 48 (2004), 61-79.

[14] Rudin, W.: Function Theory in the unit ball of $\mathbb{C}^{n}$. Fundamental Principles of Mathematical Science 241. Springer-Verlag, New York-Berlin, 1980 .

[15] Stroethoff, K.: The Berezin transform and operators on spaces of analytic functions. In Linear operators (Warsaw, 1994), 361-380. Banach Center Publ. 38. Polish Acad. Sci., Warsaw, 1997. 
[16] SuÁrez, D.: Approximation and symbolic calculus for Toeplitz algebras on the Bergman space. Rev. Mat. Iberoamericana 20 (2004), 563-610.

[17] SuÁrez, D.: Approximation and the $n$-Berezin transform of operators on the Bergman space. J. Reine Angew. Math. 581 (2005), 175-192.

[18] Zygmund, A.: Trigonometric series. Cambridge University Press, New York, 1959.

Recibido: 13 de septiembre de 2004

Revisado: 5 de abril de 2005

Kyesook Nam

Department of Mathematics

Hanshin University

Gyeonggi 447-791, KOREA

ksnam@hanshin.ac.kr

Dechao Zheng

Department of Mathematics

Vanderbilt University

Nashville, Tennessee 37240, USA

zheng@math. vanderbilt.edu

Changyong Zhong

Department of Mathematics

Vanderbilt University

Nashville, Tennessee 37240, USA

zhong@math . vanderbilt.edu

The first author was partially supported by KOSEF(R01-2003-000-10243-0). The second author was partially supported by the National Science Foundation. 